\title{
THERAPEUTICALLY EXPLORING PERSISTER METABOLISM IN BACTERIA
}

2 Sayed G. Mohiuddin ${ }^{1}$, Thuy Hoang ${ }^{1}$, Adesola Saba ${ }^{1}$, Prashant Karki ${ }^{1}$, Mehmet A. Orman ${ }^{1 *}$

$3{ }^{1}$ Department of Chemical and Biomolecular Engineering, University of Houston, Houston, TX, $4 \quad 77204$

$5 \quad$ *Corresponding Author. S222 Engineering Bldg 1, 4726 Calhoun Rd, Houston, TX 77204, and 6 Phone: 713-743-6785, Email: morman@central.uh.edu

ABSTRACT 
Conventional therapies for infectious diseases target the mechanisms that enable the rapid growth of bacterial cell populations. Although this can provide a clinical benefit, this benefit is usually short-lived for persistent and recurrent infections, and a large body of evidence suggests that small subpopulations of microbial cells invariably survive this initial selection pressure. One of the proposed mechanisms for this tolerance is via the establishment of a latent pool of persister cells (1). Persisters are an important health problem, because they are thought to underlie the propensity of recurrent infections to relapse (2-4) and serve as a reservoir from which drug-resistant mutants can emerge (5-8). Persisters exhibit a diverse range of proliferative, metabolic, and transcriptional activities. Whereas there are some variants that can grow in the presence of antibiotics, these are very rare and often survive by the drugs (10). By contrast, the most abundant variant is the type I persisters, which do not grow in the presence of antibiotics and are largely formed by passage through the stationary phase before antibiotic treatments (11). Elucidating the formation mechanisms of these preexisting, nonproliferating type I persisters is of special interest; because, these variants are found among many bacterial species, are often multidrug tolerant, and their eradication is a huge challenge $(1,3,4)$.

We previously showed that type I persisters mostly derive from stationary-phase cells with high redox activities that are maintained by endogenous protein and RNA degradation (12). We speculated that this intracellular degradation (i.e., self-digestion or autophagy) not only provides energy to bacterial cells in a non-nutritive environment, but also produces selfinflicted damage that renders the cells less fit for rapid resumption of growth. Inhibiting stationary-phase respiratory activities chemically (treatment with potassium cyanide or nitric oxide to suppress cellular respiration), environmentally (culturing under anaerobic 
conditions), or even genetically (genes encoding redox enzymes such as $u b i F, s u c B, m d h$, $a c e E, s d h C$, and $a c n B$ ) reduced persister levels by preventing digestion of endogenous proteins and RNA, yielding cells that were more capable of translation and replication and thus susceptible to cell death when exposed to antibiotics $(12,13)$. This reduction in persister levels was not found to be associated with the inhibition of RNA and protein synthesis or elimination of reactive oxygen species (ROS) (12). These results also suggest persisters harbor ETC activities associated with bacterial cytochromes, oxidoreductases and PMF, which was supported by previous studies, where "aminoglycoside (AG) potentiation assays" were used (14-16). Our current study further provides strong support for the notion that persister metabolism is a rich source of novel antipersister strategies. Using a highthroughput screening approach and a small chemical library (Biolog Phenotype Arrays containing FDA-approved drugs and antibiotics) in the current study, we identified a subset of drugs that can reduce persistence in Gram-negative bacteria by targeting their metabolism.

\section{RESULTS}

\section{Chlorpromazine pretreatment can reduce $E$. coli persistence}

As effective sterilization methods for treating chronic and recurrent infections remain scarce, identifying novel targets, together with medicinally relevant inhibitors, is becoming an urgent priority to improve the therapies for these infections. Inhibition of respiration throughout stationary phase or deletion of genes encoding TCA and ETC enzymes was shown to delay intracellular degradation and persistence by reducing cellular metabolic activity (12). ETC reactions are powered by oxidizing/reducing equivalents and are essential for ATP generation by the proton motive force (PMF). If this is the case, we should be able to reduce persister formation in stationary phase by targeting a key component, i.e., ATP synthase, in this metabolic mechanism. Chlorpromazine, which is an FDA approved 
antidepressant drug that is effective, safe and listed as an essential medicine by the World Health Organization (17), was demonstrated to inhibit the catalytic complex of rotary nanomotor ATP synthase (F1-ATPase) in E. coli cells $(18,19)$. As expected, when we treat stationary-phase cultures with chlorpromazine (Fig. 1A), at a concentration that does not affect stationary-phase cell survival (Fig. S1), we were able to reduce the stationary-phase persistence (Fig. 1BC). We note that cells were not treated with antibiotics directly in stationary-phase cultures, as normal cells are intrinsically tolerant in these cultures $(14,16)$. The stationary-phase cells were first washed to remove the metabolic inhibitors, transferred to fresh medium, and then treated with antibiotics to stimulate non-persister cell killing. Pretreatment with chlorpromazine also reduced stationary phase metabolic activities by redox sensor green (RSG) dye (Figs. 1DE). RSG can readily penetrate bacteria and yield green fluorescence when reduced by bacterial reductases; hence, fluorescent signals produced by RSG correlate with cellular metabolic activities (Fig. S2). Overall, these results verify that bacterial metabolism is a rich source of novel antipersister strategies.

\section{Chlorpromazine pretreatment reduced non-growing cell levels in stationary phase cultures}

Although some persistent infections are associated with clinically apparent chronic symptoms, some cases are asymptomatic for a long period of time (e.g., a decade) and can develop clinically significant diseases at later times (20). The bacteria causing asymptomatic infections can be present within the host system in a nonreplicating or slowly replicating state (generally referred to as "viable but non-culturable" or VBNC state) and cannot be easily cultured in vitro $(21,22)$. We and others have shown that antibiotic-treated cultures have many more VBNC cells than persisters ( 2-log-fold more) $(14,23-25)$. Both persister and VBNC cells are stained as live, retain metabolic activity, and often appear as nongrowing during the antibiotic treatment (14). The only means to distinguish these subpopulations lies in the ability of persisters but not VBNC cells to recolonize in standard 
culture media in the absence of antibiotics. To determine whether the chlorpromazine pretreatment eliminate VBNC, we used our published method where we monitor cell proliferation via an inducible fluorescent protein (mCherry) expression cassette $(12,14,26)$, in which mCherry-positive cells from late-stationary-phase cultures are inoculated in fresh medium in the absence of inducer (Fig. $1 \mathrm{~F}, \mathrm{t}=0$ ). Flow cytometry reveals ongoing cell division as a dilution of mCherry, whereas the fluorescence levels are maintained in the nonproliferating subpopulation (Fig. 1F, WT at $\mathrm{t}=2.5 \mathrm{~h}$ ). Although persisters were shown to be enriched in this subpopulation (26), most of these non-growing cells were identified as VBNC cells (26) (Fig. S3AB), which were not detected in the chlorpromazine treated cultures (Fig. 1F, Chlorpromazine at $\mathrm{t}=2.5 \mathrm{~h}$, and Fig. S3A). The reduction in both persister and VBNC cell levels in the chlorpromazine treated cultures points out these two phenotypes may be related. Consistent with the general notion in the field, it is possible that persistence may be a transitory phase leading to the VBNC state (22). Whether persistence contributes to the accumulation of VBNC cells due to the catabolism of intracellular components warrants further investigation.

\section{High-throughput screening detected chemical compounds that target $E$. coli metabolism and}

\section{persistence}

To directly measure protein degradation rates in stationary-phase cultures, we previously developed an assay using GFP that is linked to a short peptide degradation tag (11 amino acid residues), ssrA, to mark it for degradation by cellular proteases, mainly ClpAP and ClpXP (Lon, Tsp and FtsH are also known to target the ssrA sequence) (27-30). Although we note that self-digestion is a complex network orchestrated by many degradative enzymes (proteases, RNases and toxins), chlorpromazine treatment suppressed degradation of this tag in stationary-phase cultures (Fig. 2A, and Fig. S4A), potentially by reducing stationaryphase cell metabolic activities (Fig. 1DE). To test whether this straightforward system can 
identify additional antipersister therapeutics, we used a small library (Biolog Phenotype Arrays), containing antibiotics and other FDA approved drugs among 360 known chemical compounds in 96-well plate formats. Cells expressing ssrA-tagged GFP were transferred to the phenotype arrays without inducer at early-stationary phase, and cultured under the conditions studied here (Fig. 2B, see Materials and Methods). GFP levels were monitored using a plate reader, with cells cultured in the presence of the solvent serving as the negative controls, and those with chlorpromazine as a positive control. Our data verify that GFP in negative-control wells is degraded within $4 \mathrm{~h}$ (Fig. S4AB). The Z-factor (predicted by analysis of test plates with negative and positive controls, as described in Materials and Methods) was calculated to be 0.836 , which indicates the robustness of our methodology (31). We employed a widely used Z-score method (calculated from the mean and the standard deviation of all measurements within the plate) (32) to determine initial hits. An absolute Z-score of $\geq 2$ is the threshold for hit detection (32). Given that each plate contains four different concentrations for each compound (information on these concentrations not disclosed by the company), the initial hits were selected among the chemicals that successfully inhibited GFP degradation (Z-score $\geq 2$ ) with at least two different concentrations (Fig. 2C and Fig. S4C). As expected, chlorpromazine, which is one of the 360 chemical compounds tested, was identified as a positive hit, verifying that our method can detect potential metabolic inhibitors (Fig. 2C). To determine chemical inhibitors that specifically target persister metabolism, the identified hits were further analyzed in additional rounds of screening to determine concentrations that lead to complete inhibition of GFP degradation without affecting the stationary-phase-cell viability (Fig. 2D, and Fig. S5 and S6). We identified that CCCP, polymyxin B, poly-L-lysine, thioridazine, and trifluoperazine did not drastically affect the cell viability at the inhibitory concentrations for GFP degradation (Fig. S6), and four drugs, except CCCP, were able to reduce persistence 
(Fig. 2E and Fig. S7). Both thioridazine and trifluoperazine fall under the category of phenothiazine antipsychotic drugs, which are tricyclic compounds structurally similar to

\section{The identified drugs can reduce Pseudomonas aeruginosa persistence}

3AB). Treating the stationary phase cells with these four chemicals further reduces VBNC formation (Fig. 3C and Fig. S8), consistent with the results obtained from chlorpromazine treatments. Overall, these results strongly support that the identified drugs eliminate bacterial persistence by inhibiting stationary phase metabolism.

Our previous results indicate that persistence is facilitated by a self-digestion mediated metabolic futile cycle, wherein energy derived from catabolism is dissipated through continuous degradation of cellular components $(12,13)$. This process also introduces a selfinflected damage in the cells that transiently repressed the cellular functions targeted by antibiotics (12). The identification and characterization of the main components of this metabolic cycle may provide a global treatment approach as it can be an evolutionarily conserved process that may occur in many prokaryotes and eukaryotes and enable survival under stressful conditions (such as nutrient depletion, aging and overpopulation) via the recycling of essential energy molecules. When we similarly tested the identified chemicals on Pseudomonas aeruginosa (PAO1), we were able to substantially reduce $P$. aeruginosa persistence, suggesting the existence of similar mechanisms in other bacteria (Fig. 3D and 
Fig. S9). These results provide further clinical relevance for the identified drugs, since $P$. aeruginosa is involved in many hospital-related biofilm infections and the predominant cause of morbidity and mortality in cystic fibrosis patients with compromised immune systems $(38-40)$.

\section{DISCUSSION}

As antibiotics are most effective against growing bacteria, the resistance of persisters has been attributed to transient growth inhibition. Experimental evidence supporting this hypothesis was obtained in 2004 by Balaban and colleagues, who showed bacteria that failed to replicate prior to an ampicillin challenge also failed to lyse or grow during antibiotic treatment, but began replicating once the antibiotic was removed (11). This seminal study led to the model that persistence is a dormant phenotype, characterized by a depressed metabolism. However, recent evidence suggests persisters can harbor electron transport chain (ETC) activities associated with bacterial cytochromes and oxidoreductases (12). They can consume certain carbon sources to generate proton motive force (PMF) (14, 15), maintain high ATP levels (41), and drive the futile production and degradation of RNA, leading to energy generation and dissipation (42). Interestingly, most persister-related genes identified so far either directly or indirectly modulate cell metabolism.

Although metabolic processes and persistence in bacteria are known to be closely related, the specific mechanisms that link these remain unknown. Our previous results indicate that self-digestion may be this link (12). The role of metabolism is significant for bacteria, because they must produce large amounts of energy and biosynthetic precursors to meet the metabolic demands of their rapid growth. This results in a number of metabolic stresses, including nutrient starvation, hypoxia, and oxidative stress, which promote intracellular degradation/damage that may transiently repress the cellular functions targeted by antibiotics. Using transmission electron microscopy (TEM) and classic starvation 
conditions to create VBNC cells, Kim et al. showed that prolonged nutrient deprivation (7

weeks) results in cells that are spherical, have an empty cytosol (due intracellular degradation), and fail to resuscitate (43). Although nutrient deprivation initially increased persister levels in their experiments, continuous intracellular degradation eventually converted most of the cells to VBNCs. Persistence may, in fact, represent a transitory phase leading to the VBNC state and contribute to accumulation of VBNC cells due to intracellular degradation. Many persistence mechanisms identified so far involve stress-related responses, which generally induce, or are associated with, cellular self-digestion (44).

Although our previous results provided evidence that intracellular degradation transiently induces persistence (12), knowledge regarding what unique metabolic mechanisms are involved is lacking. Our current and previous results indicate that, despite their nonproliferating state, persister cells still exist in a metabolic steady state, where energy is continually produced and consumed (12-14). Our results further showed that targeting persister metabolism holds great potential for eradicating these dangerous phenotypes, as verified by the identified drugs (i.e., chlorpromazine, thioridazine, trifluoperazine, polymyxin B and poly-L-lysine), which are already known to target bacterial redox activities (45). Chlorpromazine (CPZ), thioridazine (TDZ), and trifluoperazine (TFP) are commonly known as first generation antipsychotic/neuroleptic drugs (45-47). Since they are the derivative of a heterocyclic phenothiazine, their mechanism of action is similar (45). The effectiveness of these drugs depends upon the ability to block dopamine receptors as the excessive dopamine is the main culprit of schizophrenia and other psychotic diseases (48). These drugs were also shown to have antimicrobial activities. In Mycobacterium tuberculosis, phenothiazines inhibit cellular respiration, leading to depletion of ATP as well as the reduction of $\mathrm{NADH} / \mathrm{NAD}+$ and menaquinol/menaquinone ratios (33-35). Because of 
their ability to inhibit bacterial efflux pumps, they were also shown to enhance the sensitivity of Staphylococcus aureus to beta-lactam antibiotics $(49,50)$.

Studies have shown that poly-1-lysine, which is a cationic polymer, can result in change of morphology in bacteria (37). In addition, treatment with poly-l-lysine raises the electric conductivity of the bacterial cells which leads to possible disruption of the cytoplasmic membrane (51). Similarly, polymyxins consist of a polypeptide cationic ring made up of 8 to 10 amino acids, which have a disruptive physiochemical effect resulting in alternation of membrane permeability in bacteria (52). In addition, type II NADH-quinone oxidoreductases, which are integral part of electron transport chain, has also been shown to be a secondary target sites of cationic peptides (53). Polymyxins have been administered for urinary tract infection, pneumonia, bacteremia, postoperative wound infections, abscesses, osteomyelitis (when given as an irrigation), and endocarditis (52).

Overall, we presented here a methodology that has been designed to challenge paradigms regarding metabolic dormancy in persisters, shed light on the often-overlooked metabolic processes of persister cells, develop a screening approach to identify metabolic inhibitors among a small library with FDA approved compounds, and integrate all proposed work to accelerate development of antipersister adjuvant therapies. Given that the cytotoxicity, cell permeability, solubility, and safety properties of FDA compounds have been well studied and documented during their preclinical and clinical research phases, discovering antipersister drugs among such libraries will have an enormous impact, because it will identify potential therapeutics that do not require the long laborious FDA approval process. Our preliminary studies have already identified a subset of drugs that can eliminate persisters even in stationary phase cultures, which represent notoriously challenging conditions for the elimination of persisters. 


\section{MATERIALS AND METHODS}

\section{Bacterial Strains and Plasmids}

Escherichia coli MG1655 wild-type (WT) and MO strains as well as pQE-80L plasmids

\section{Media, Chemicals and Culture Conditions}

All chemicals were purchased from Fisher Scientific (Atlanta, GA), VWR International (Pittsburg, PA) or Sigma Aldrich (St. Louis, MO). Luria-Bertani (LB) liquid media, prepared from its components $(5 \mathrm{~g}$ yeast extract, $10 \mathrm{~g}$ tryptone and $10 \mathrm{~g}$ sodium chloride in $1 \mathrm{~L}$ ultra-pure DI water), and Mueller-Hinton (MH) liquid media (21 g premixed $\mathrm{MH}$ in 1 L ultra-pure DI water) were used to grow E. coli and $P$. aeruginosa, respectively $(12,55$, 56). LB agar media (40 g premixed LB agar in $1 \mathrm{~L}$ ultra-pure DI water) and MH agar media (38 g premixed $\mathrm{MH}$ agar in $1 \mathrm{~L}$ ultra-pure DI water) were used to enumerate the colony forming units (CFUs) of E. coli and P. aeruginosa strains, respectively. Phosphate Buffered Saline (PBS) solution was used to wash the cells to remove the chemicals and antibiotics 
before plating them on agar media. Concentrations of $5 \mu \mathrm{g} / \mathrm{mL}$ ofloxacin and $200 \mu \mathrm{g} / \mathrm{ml}$ ampicillin were used for persister assays (12). MIC ranges for E. coli MG1655 were found to be $3.125-6.25 \mu \mathrm{g} / \mathrm{ml}$ for ampicillin and $0.039-0.078 \mu \mathrm{g} / \mathrm{ml}$ for ofloxacin by using a method based on serial 2-fold dilutions of antibiotics in $2 \mathrm{ml} \mathrm{LB}$ media in $14 \mathrm{ml}$ test tubes (13). MIC range for $P$. aeruginosa (PA01) were found to be $0.3125-0.625 \mu \mathrm{g} / \mathrm{ml}$ for ofloxacin. For selection and retention of plasmids in bacteria, $50 \mu \mathrm{g} / \mathrm{ml}$ kanamycin was added in culture media (12). To induce fluorescent protein expression, $1 \mathrm{mM}$ IPTG was used (12). Primary drug screening was performed using Phenotype MicroArrays (PM1120) in 96-well plate formats, containing various chemicals including FDA approved compounds (Biolog Inc., Hayward, CA). Eleven chemicals, identified as initial hits, were purchased separately for further investigation: amitriptyline hydrochloride (Fisher catalog\#50-144-4347), trifluoperazine hydrochloride (Fisher catalog\#T28495G), thioridazine hydrochloride (Fisher catalog\#30-705-0), chlorpromazine hydrochloride (Fisher catalog\#C24815G), CCCP (Fisher catalog\# 04-525-00), protamine sulfate (Fisher catalog\# AAJ6292609), promethazine hydrochloride (Fisher catalog\#P2029100G), dodecyltrimethyl ammonium bromide (Fisher catalog\# D146825G), triclosan (Fisher catalog\# 64-795-01GM), polymyxin B Sulfate (Fisher catalog\# 52-915-GM) and poly-Llysine hydrochloride (VWR catalog\# IC15269080). All chemicals were dissolved in ultrapure DI water followed by filter-sterilization, except for CCCP and triclosan which were dissolved in DMSO. All LB and MH media were sterilized by autoclaving. Overnight precultures were prepared in 14-ml falcon tube containing $2 \mathrm{ml} \mathrm{LB}$ broth inoculated from a $25 \%$ glycerol $\left(-80{ }^{\circ} \mathrm{C}\right)$ cells stock and grown for $24 \mathrm{~h}$ at $37{ }^{\circ} \mathrm{C}$ with shaking $(250 \mathrm{rpm})$. Overnight pre-cultures were diluted in fresh $2 \mathrm{ml}$ media in 14-ml test tubes or $25 \mathrm{ml}$ media in 250-ml baffled flasks for the subsequent assays as describe below. Cells cultured in the 
presence of the solvent (DI water or DMSO) served as controls when the cultures were treated with chemical inhibitors.

\section{9}

\section{Cell Growth and Persister Assays}

Overnight pre-cultures were diluted 1000-fold in $2 \mathrm{ml}$ fresh LB media in test tubes and grown at $37{ }^{\circ} \mathrm{C}$ with shaking. Cell growth was monitored up to 24 hours by measuring optical density at $600 \mathrm{~nm}$ wavelength $\left(\mathrm{OD}_{600}\right)$ with a plate reader (Varioskan LUX Multimode Microplate Reader, ThermoFisher, Waltham, MA) for selected time points. Cells were treated with chemicals at early-stationary $(t=5 \mathrm{~h})$ when required. At latestationary phase ( $\mathrm{t}=24 \mathrm{~h}$ ), cells were diluted in $2 \mathrm{ml}$ fresh media (yielding $\sim 5 \times 10^{7}$ cells $/ \mathrm{ml}$ ) with antibiotics (5 $\mu \mathrm{g} / \mathrm{ml}$ ofloxacin or $200 \mu \mathrm{g} / \mathrm{ml}$ ampicillin) in test tubes and incubated at $37^{\circ} \mathrm{C}$ with shaking. At designated time points $(\mathrm{t}=0,1,2,3,4,5$ and $6 \mathrm{~h}), 100 \mu 1$ samples were collected and washed with PBS to dilute the antibiotics to sub-MIC levels, followed by resuspension in $100 \mu \mathrm{l}$ of PBS. Ten microliters of the cell suspension were serially diluted and plated on LB agar media to enumerate CFUs. The remaining $90 \mu \mathrm{l}$ cell suspensions were also plated to increase the limit of detection. The agar plates were incubated at $37^{\circ} \mathrm{C}$ for $16 \mathrm{~h}$, which was found to be sufficient for $E$. coli colony formation (data not shown). The procedures described above were repeated using 250 ml-baffled flasks with $25 \mathrm{ml}$ media to determine the effects of culture volume, aeration and mixing on cell growth and persistence. We did not see significant differences between the results of flask and test tube experiments (data not shown).

\section{Redox Sensor Green Dye Staining}

Stationary-phase reductase and electron transport chain activities were measured with Redox Sensor Green (RSG) dye (ThermoFisher, catalog\# B34954) according to manufacturer's instructors. Cells at late-stationary phase $(\mathrm{t}=24 \mathrm{~h})$ were diluted 100 -fold in $1 \mathrm{ml}$ PBS in flow cytometry tubes ( $5 \mathrm{ml}$ round bottom falcon tubes, size: $12 \times 75 \mathrm{~mm})$ and 
stained with RSG at $1 \mu \mathrm{M}$ concentration. For negative controls, CCCP $(10 \mu \mathrm{M})$ was added in the cell suspensions 5 minutes before RSG staining to disrupt membrane electron transport. Mid-exponential-phase cells were used as positive controls $(26,43)$. Samples were incubated at $37^{\circ} \mathrm{C}$ for 10 minutes before analyzing with a flow cytometer (NovoCyte Flow Cytometer, NovoCyte 3000RYB, ACEA Biosciences Inc., San Diego, CA). Forward and side scatter parameters of unstained controls were used to gate the cell populations on flow diagrams. Cells were excited at $488 \mathrm{~nm}$ with solid-state laser, and green fluorescence was collected with a 530/30 bandpass filter. To analyze the effect of chemical inhibitors (e.g., chlorpromazine) on stationary-phase-cell metabolism, cells at early-stationary phase $(\mathrm{t}=5 \mathrm{~h}$ ) were treated with the chemicals at indicated concentrations, and RSG staining was performed at $\mathrm{t}=24 \mathrm{~h}$ as described above.

\section{Monitoring Cell Division and Quantifying VBNC Cells}

To monitor cell division and quantify non-growing cell subpopulations, inducible fluorescent protein (mCherry) expression systems were used. Overnight pre-cultures of $E$. coli $\mathrm{MO}$ strain were diluted 1000 -fold in $2 \mathrm{ml}$ LB media with $1 \mathrm{mM}$ IPTG (to induce $m$ Cherry) in test tubes and grown as described. We previously showed that mCherry expression cassette or overexpressing mCherry did not affect the $E$. coli persistence $(12,14$, 26). If necessary, cells at early-stationary phase $(\mathrm{t}=5 \mathrm{~h})$ were treated with chemical inhibitors (e.g., chlorpromazine) at indicated concentrations. At $\mathrm{t}=24 \mathrm{~h}$, mCherry-positive cells were collected, washed twice with PBS to remove the IPTG, resuspended in $25 \mathrm{ml}$ fresh LB media without inducer in $250 \mathrm{ml}$ baffled flasks and cultured at $37{ }^{\circ} \mathrm{C}$ with shaking. At designated time points ( $t=0,1,2$, and $2.5 \mathrm{~h}$ ), cells were collected, washed and resuspended in PBS to measure their fluorescent protein content with a flow cytometer. When necessary, cells were further diluted in PBS to reach a desired cell density for the flow analysis $\left(10^{6}-10^{7}\right.$ per $\left.\mathrm{ml}\right)$. Cell division was monitored by measuring the dilution rate of fluorescent protein at single 
cell level. At $\mathrm{t}=0 \mathrm{~h}$, all cells exhibited a high level of red fluorescence, which declined as the cells divided, except in a small subpopulation whose fluorescence remained constant due to the lack of division $(\mathrm{t}=2.5 \mathrm{~h})$. Given that ampicillin only targets the proliferating cells, the cultures were further challenged with ampicillin $(200 \mu \mathrm{g} / \mathrm{ml})$ to quantify VBNC and persister cells in non-growing cell subpopulations. Using LIVE/DEAD staining, FACS and clonogenic survival assays, we previously showed that antibiotic sensitive cells were rapidly lysed by ampicillin while VBNC and persister cells remained intact (14). The intact cells were quantified using the volumetric-based cell counting feature of the NovoCyte Flow Cytometer. Persisters were quantified by enumerating the CFUs after plating the ampicillin treated cultures as described above. Intact cells that did not form colonies on standard medium were classified as VBNC cells $(14,21-25)$. All samples were assayed with lasers emitting at $561 \mathrm{~nm}$ and red fluorescence was collected by $615 / 20 \mathrm{~nm}$ bandpass filter.

\section{Fluorescent Protein Degradation Assay}

Overnight pre-cultures of E. coli MG1655 harboring pQE-80L-ssrA-gfp were inoculated $(1: 1000)$ in $2 \mathrm{ml} \mathrm{LB}$ in test tubes, grown in the presence of IPTG (to induce $s s r A$ tagged $g f p)$ until the early stationary phase $(\mathrm{t}=5 \mathrm{~h})$. Then, the cells were washed to remove the inducer, resuspended in filter-sterilized $2 \mathrm{ml}$ spent medium (obtained from cultures grown under identical conditions without the inducer) and cultured in test tubes at $37{ }^{\circ} \mathrm{C}$ with shaking. When necessary, cell suspensions were treated with chemical inhibitors. At designated time points, $200 \mu 1$ samples were collected to measure their GFP levels with a plate reader. Excitation and emission wavelengths for GFP detection was $485 \mathrm{~nm}$ and 511 $\mathrm{nm}$, respectively.

\section{Chemical Screening}

Early-stationary-phase cells expressing ssrA-tagged GFP (grown in $25 \mathrm{ml}$ LB with IPTG in $250 \mathrm{ml}$ baffled flasks) were washed, resuspended in spent medium (without inducer), 
transferred to 96 -well PM plates $(100 \mu$ l per well) containing the chemical library, covered with sterile, oxygen-permeable sealing membranes, and cultured in a shaker at $37{ }^{\circ} \mathrm{C}$ and $250 \mathrm{rpm}$. GFP levels were monitored for $4 \mathrm{~h}$ (which was found to be sufficient) using a plate reader, with cells cultured in the presence of the solvent serving as the negative controls, and those with chlorpromazine as a positive control. GFP measurements taken at $4 \mathrm{~h}$ were normalized to those taken at $0 \mathrm{~h}$ to eliminate any variations in initial cell concentrations. Zscore method, calculated from the mean and the standard deviation of all measurements within the plate (31) was used to determine initial hits:

$$
Z-\text { score }=\frac{X_{i}-\bar{X}}{S_{X}}
$$

where $X_{i}$ is the measurement (normalized) of the $i^{\text {th }}$ compound, $\bar{X}$ and $S_{X}$ are the mean and the standard deviation of all measurements. An absolute Z-score of $\geq 2$, which correlates to a P-value of 0.045 (32), was assumed to be the threshold for hit detection. We note that each plate contains four different concentrations for each compound (information on these concentrations not disclosed by the company). Z-scores were calculated for each concentration set. The initial hits were selected among the chemicals that successfully inhibited GFP degradation (Z-score $\geq 2$ ) with at least two different concentrations.

Assay validation was evaluated by Z-factor calculated from the mean and standard deviation values of the positive (p) and the negative (n) control plates, as follows:

$$
Z-\text { factor }=1-3 \times \frac{\left(S_{p}+S_{n}\right)}{\left|\bar{X}_{p}-\bar{X}_{n}\right|}
$$




\section{Validating the Selected Chemicals}

To fully assess their utility and effectiveness, the selected chemical hits were analyzed at various concentrations with the aforementioned assays. Overnight pre-cultures of E. coli strains (WT, MO or cells expressing ssrA tagged $g f p$ ) were inoculated (1:1000) in $2 \mathrm{ml}$ LB (IPTG added for the cells harboring inducible fluorescent proteins) in test tubes and cultured as described. Cells at $\mathrm{t}=5 \mathrm{~h}$ were treated with chemicals at indicated concentrations. Fluorescent protein degradation assays throughout the stationary phase after the treatments were performed for the cultures of E. coli cells expressing SsrA tagged GFP; persister and cell survival assays at late-stationary phase $(\mathrm{t}=24 \mathrm{~h})$ were performed for WT cultures; and finally, cell division assays at late-stationary phase were performed for the E. coli MO cultures.

\section{Pseudomonas aeruginosa Persister Assay}

Overnight pre-cultures of Pseudomonas aeruginosa (PA01) were inoculated (1:1000) in 2 $\mathrm{ml} \mathrm{MH} \mathrm{broth} \mathrm{in} \mathrm{test} \mathrm{tubes} \mathrm{and} \mathrm{cultured} \mathrm{as} \mathrm{described} \mathrm{before.} \mathrm{At} \mathrm{early} \mathrm{stationary} \mathrm{phase} \mathrm{(} \mathrm{t}=5$ h), cells were treated with chemicals at indicated concentrations. At $t=24 \mathrm{~h}$, cells were washed to remove chemicals and inoculated (1:100) in $1 \mathrm{ml} \mathrm{MH}$ broth followed by ofloxacin $(5 \mu \mathrm{g} / \mathrm{ml})$ treatment. At $\mathrm{t}=0$ (before ofloxacin treatments), ten microliter cell suspensions were serially diluted and spotted on MH agar media to enumerate initial CFUs, which enables us to assess the impacts of chemical treatments on P. aeruginosa (PA01) cell viability. To enumerate persister levels at $\mathrm{t}=6 \mathrm{~h}$, ofloxacin treated cultures were washed, serially diluted and plated on $\mathrm{MH}$ agar media to incubate $20 \mathrm{~h}$ at $37{ }^{\circ} \mathrm{C}$. Twenty-hour incubation was found to be sufficient for P. aeruginosa (PA01) colony formation (data not shown). 


\section{8}

\section{Statistical analysis}

Two tailed t-test with unequal variances was used to evaluate the statistical significance, where $\mathrm{P}<0.05$ (55). At least three independent biological replicate was performed for all experiments. All data points on linear-scale graphs indicate mean value \pm standard error; however, for logarithmic-scale graphs, standard deviations were used to better represent the error bars.

\section{REFERENCES}

1. van den Bergh B, Fauvart M, Michiels J. 2017. Formation, physiology, ecology, evolution and clinical importance of bacterial persisters. FEMS Microbiol Rev.

2. Fauvart M, de Groote VN, Michiels J. 2011. Role of persister cells in chronic infections: Clinical relevance and perspectives on anti-persister therapies. J Med Microbiol.

3. Lewis K. 2007. Persister cells, dormancy and infectious disease. Nat Rev Microbiol.

4. Lewis K. 2010. Persister Cells. Annu Rev Microbiol 64:357-372.

5. Windels EM, Michiels JE, Fauvart M, Wenseleers T, Van den Bergh B, Michiels J. 2019. Bacterial persistence promotes the evolution of antibiotic resistance by increasing survival and mutation rates. ISME J 13:1239-1251.

6. Van Den Bergh B, Michiels JE, Wenseleers T, Windels EM, Boer P Vanden, Kestemont D, De Meester L, Verstrepen KJ, Verstraeten N, Fauvart M, Michiels J. 2016. Frequency of antibiotic application drives rapid evolutionary adaptation of Escherichia coli persistence. Nat Microbiol 1:1-7.

7. Levin-Reisman I, Ronin I, Gefen O, Braniss I, Shoresh N, Balaban NQ. 2017. Antibiotic tolerance facilitates the evolution of resistance. Science (80- ) 355:826-830.

8. Barrett TC, Mok WWK, Murawski AM, Brynildsen MP. 2019. Enhanced antibiotic 
9. $\quad \mathrm{Pu}$ Y, Zhao Z, Li Y, Zou J, Ma Q, Zhao Y, Ke Y, Zhu Y, Chen H, Baker MAB, Ge H, Sun

13. Orman MA, Brynildsen MP. 2016. Persister formation in Escherichia coli can be inhibited by treatment with nitric oxide. Free Radic Biol Med 93:145-154.

14. Orman MA, Brynildsen MP. 2013. Establishment of a Method To Rapidly Assay Bacterial Persister Metabolism. Antimicrob Agents Chemother 57:4398-4409.

15. Allison KR, Brynildsen MP, Collins JJ. 2011. Metabolite-enabled eradication of bacterial persisters by aminoglycosides. Nature 473:216-220.

16. Mok WWK, Orman MA, Brynildsen MP. 2015. Impacts of Global Transcriptional Regulators on Persister Metabolism. Antimicrob Agents Chemother 59:2713-2719.

17. WHO. 2018. WHO Model Lists of Essential Medicines. WHO. World Health Organization.

18. Bullough DA, Kwan M, Laikind PK, Yoshida M, Allison WS. 1985. The varied responses of different F1-ATPases to chlorpromazine. Arch Biochem Biophys 236:567-575. 
464 19. Chazotte B, Vanderkooi G, Chignell D. 1982. Further studies on F1-ATPase inhibition by local anesthetics. BBA - Bioenerg 680:310-316.

20. Grant SS, Hung DT. 2013. Persistent bacterial infections, antibiotic tolerance, and the oxidative stress response. Virulence.

21. Oliver JD. 2010. Recent findings on the viable but nonculturable state in pathogenic bacteria. FEMS Microbiol Rev.

22. Ayrapetyan M, Williams TC, Oliver JD. 2015. Bridging the gap between viable but nonculturable and antibiotic persistent bacteria. Trends Microbiol.

23. Jõers A, Kaldalu N, Tenson T. 2010. The frequency of persisters in Escherichia coli reflects the kinetics of awakening from dormancy. J Bacteriol 192:3379-3384.

24. Luidalepp H, Jõers A, Kaldalu N, Tenson T. 2011. Age of inoculum strongly influences persister frequency and can mask effects of mutations implicated in altered persistence. $\mathrm{J}$ Bacteriol 193:3598-3605.

25. Roostalu J, Jõers A, Luidalepp H, Kaldalu N, Tenson T. 2008. Cell division in Escherichia coli cultures monitored at single cell resolution. BMC Microbiol 8.

26. Orman MA, Brynildsen MP. 2013. Dormancy Is Not Necessary or Sufficient for Bacterial Persistence. Antimicrob Agents Chemother 57:3230-3239.

27. Choy JS, Aung LL, Karzai AW. 2007. Lon protease degrades transfer-messenger RNAtagged proteins. J Bacteriol 189:6564-6571.

28. Herman C, Thévenet D, Bouloc P, Walker GC, D’Ari R. 1998. Degradation of carboxyterminal-tagged cytoplasmic proteins by the Escherichia coli protease HflB (FtsH). Genes Dev 12:1348-1355.

29. Spiers A, Lamb HK, Cocklin S, Wheeler KA, Budworth J, Dodds AL, Pallen MJ, Maskell 
DJ, Charles IG, Hawkins AR. 2002. PDZ domains facilitate binding of high temperature requirement protease $\mathrm{A}(\mathrm{HtrA})$ and tail-specific protease (Tsp) to heterologous substrates through recognition of the small stable RNA A (ssrA)-encoded peptide. J Biol Chem 277:39443-39449.

30. Flynn JM, Levchenko I, Seidel M, Wickner SH, Sauer RT, Baker TA. 2001. Overlapping recognition determinants within the ssrA degradation tag allow modulation of proteolysis. Proc Natl Acad Sci 98:10584-10589.

31. Malo N, Hanley JA, Cerquozzi S, Pelletier J, Nadon R. 2006. Statistical practice in highthroughput screening data analysis. Nat Biotechnol.

32. Martin HL, Adams M, Higgins J, Bond J, Morrison EE, Bell SM, Warriner S, Nelson A, Tomlinson DC. 2014. High-content, high-throughput screening for the identification of cytotoxic compounds based on cell morphology and cell proliferation markers. PLoS One 9:1-8.

33. Yano T, Lin-Sheng L, Weinstein E, Teh JS, Rubin H. 2006. Steady-state kinetics and inhibitory action of antitubercular phenothiazines on Mycobacterium tuberculosis Type-II NADH-menaquinone oxidoreductase (NDH-2). J Biol Chem 281:11456-11463.

34. Weinstein EA, Yano T, Li L-S, Avarbock D, Avarbock A, Helm D, McColm AA, Duncan K, Lonsdale JT, Rubin H. 2005. Inhibitors of type II NADH:menaquinone oxidoreductase represent a class of antitubercular drugs. Proc Natl Acad Sci U S A 102:4548-4553.

35. Boshoff HIM, Myers TG, Copp BR, McNeil MR, Wilson MA, Barry CE. 2004. The Transcriptional Responses of Mycobacterium tuberculosis to Inhibitors of Metabolism. J Biol Chem 279:40174-40184.

36. Domingues MM, Inácio RG, Raimundo JM, Martins M, Castanho MARB, Santos NC. 2012. Biophysical characterization of polymyxin B interaction with LPS aggregates and 
membrane model systems. Biopolymers 98:338-344.

37. Li YQ, Han Q, Feng JL, Tian WL, Mo HZ. 2014. Antibacterial characteristics and mechanisms of e \{open\}-poly-lysine against Escherichia coli and Staphylococcus aureus. Food Control 43:22-27.

38. Lyczak JB, Cannon CL, Pier GB. 2000. Establishment of Pseudomonas aeruginosa infection: lessons from a versatile opportunist. Microbes Infect 2:1051-1060.

39. Stover CK, Pham XQ, Erwin AL, Mizoguchi SD, Warrener P, Hickey MJ, Brinkman FS, Hufnagle WO, Kowalik DJ, Lagrou M, Garber RL, Goltry L, Tolentino E, WestbrockWadman S, Yuan Y, Brody LL, Coulter SN, Folger KR, Kas A, Larbig K, Lim R, Smith K, Spencer D, Wong GK, Wu Z, Paulsen IT, Reizer J, Saier MH, Hancock RE, Lory S, Olson M V. 2000. Complete genome sequence of Pseudomonas aeruginosa PAO1, an opportunistic pathogen. Nature 406:959-964.

40. Mulcahy LR, Burns JL, Lory S, Lewis K. 2010. Emergence of Pseudomonas aeruginosa strains producing high levels of persister cells in patients with cystic fibrosis. J Bacteriol 192:6191-6199.

41. Baidoo EEK, Weaver D, Bokinsky G, Garcia-Martin H, Keasling JD, Lee TS, Burd H, Alonso-Gutierrez J, Akella S. 2013. HipA-Triggered Growth Arrest and -Lactam Tolerance in Escherichia coli Are Mediated by RelA-Dependent ppGpp Synthesis. J Bacteriol 195:3173-3182.

42. Mok WWK, Park JO, Rabinowitz JD, Brynildsen MP. 2015. RNA futile cycling in model persisters derived from mazF accumulation. MBio 6:1-13.

43. Kim JS, Chowdhury N, Yamasaki R, Wood TK. 2018. Viable but non-culturable and persistence describe the same bacterial stress state. Environ Microbiol 20:2038-2048.

44. Nyström T. 2004. Stationary-Phase Physiology. Annu Rev Microbiol 58:161-181. 
45. Ohlow MJ, Moosmann B. 2011. Phenothiazine: The seven lives of pharmacology's first lead structure. Drug Discov Today.

46. Dudley K, Liu X, De Haan S. 2017. Chlorpromazine dose for people with schizophrenia. Cochrane Database Syst Rev. John Wiley and Sons Ltd.

47. Fenton M, Rathbone J, Reilly J, Sultana A. 2007. Thioridazine for schizophrenia. Cochrane Database Syst Rev. John Wiley and Sons Ltd.

48. Girault JA, Greengard P. 2004. The Neurobiology of Dopamine Signaling, p. 641-644. In Archives of Neurology.

49. Amaral L, Viveiros M. 2012. Why thioridazine in combination with antibiotics cures extensively drug-resistant Mycobacterium tuberculosis infections. Int J Antimicrob Agents. Elsevier B.V.

50. Thorsing M, Klitgaard JK, Atilano ML, Skov MN, Kolmos HJ, Filipe SR, Kallipolitis BH. 2013. Thioridazine Induces Major Changes in Global Gene Expression and Cell Wall Composition in Methicillin-Resistant Staphylococcus aureus USA300. PLoS One 8:1-13.

51. Ye R, Xu H, Wan C, Peng S, Wang L, Xu H, Aguilar ZP, Xiong Y, Zeng Z, Wei H. 2013. Antibacterial activity and mechanism of action of \$E\$-poly-1-lysine. Biochem Biophys Res Commun 439:148-153.

52. Evans ME, Feola DJ, Rapp RP. 1999. Polymyxin B sulfate and colistin: Old antibiotics for emerging multiresistant gram-negative bacteria. Ann Pharmacother.

53. Deris ZZ, Akter J, Sivanesan S, Roberts KD, Thompson PE, Nation RL, Li J, Velkov T. 2014. A secondary mode of action of polymyxins against Gram-negative bacteria involves the inhibition of NADH-quinone oxidoreductase activity. J Antibiot (Tokyo) 67:147-151.

54. Weichart D, Querfurth N, Dreger M, Hengge-Aronis R. 2003. Global role for ClpP- 
containing proteases in stationary-phase adaptation of Escherichia coli. J Bacteriol $185: 115-125$.

55. Amato S, Orman M, Brynildsen M. 2013. Metabolic Control of Persister Formation in Escherichia coli. Mol Cell 50:475-487.

56. Keren I, Kaldalu N, Spoering A, Wang Y, Lewis K. 2004. Persister cells and tolerance to antimicrobials. FEMS Microbiol Lett 230:13-18.

57. Zhang JH, Chung TDY, Oldenburg KR. 1999. A simple statistical parameter for use in evaluation and validation of high throughput screening assays. J Biomol Screen 4:67-73.

58. Keren I, Kaldalu N, Spoering A, Wang Y, Lewis K. 2004. Persister cells and tolerance to antimicrobials. FEMS Microbiol Lett 230:13-18.

\section{ACKNOWLEDGEMENTS}

We would like to thank Dr. Aslan Massahi for providing assistance in persister assays.

Funding. The research was supported by NIH/NIAID K22AI125468 Career Transition award and University of Houston start up grant.

Author Contributions. S.G.M, T.H, A.S., P.K. and M.A.O. conceived and designed the study. S.G.M, T.H, A.S. and P.K. performed the experiments. S.G.M., T.H, A.S., P.K. and M.A.O. analyzed the data and wrote the paper.

Competing Interests. The authors declare no competing interests.

Data and materials availability. Data provided in this paper including supplementary materials are sufficient to assess the findings of this paper. Additional data of this paper can be obtained upon request. 
A

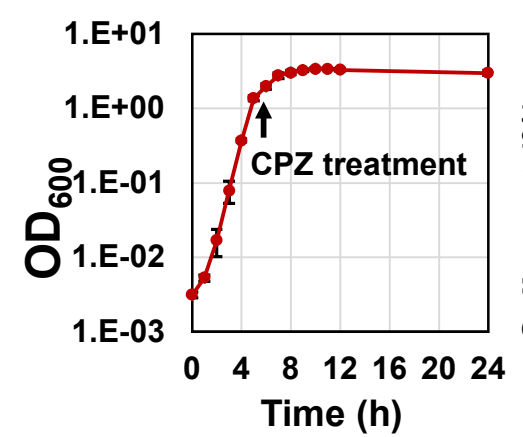

B

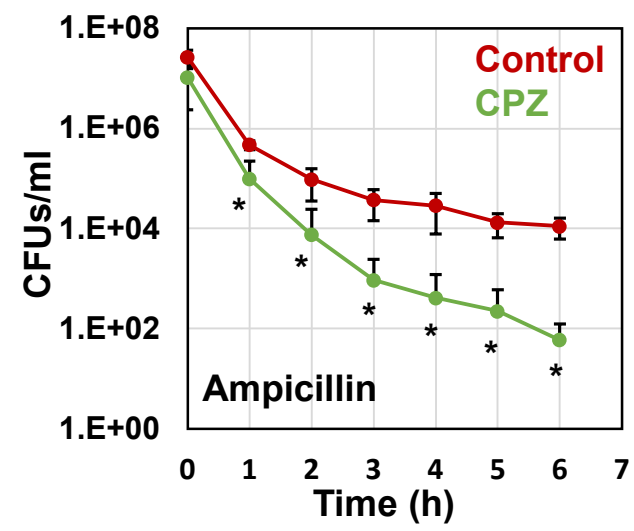

D $\quad$ E

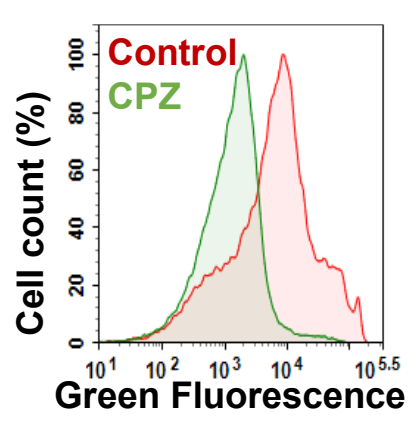

C
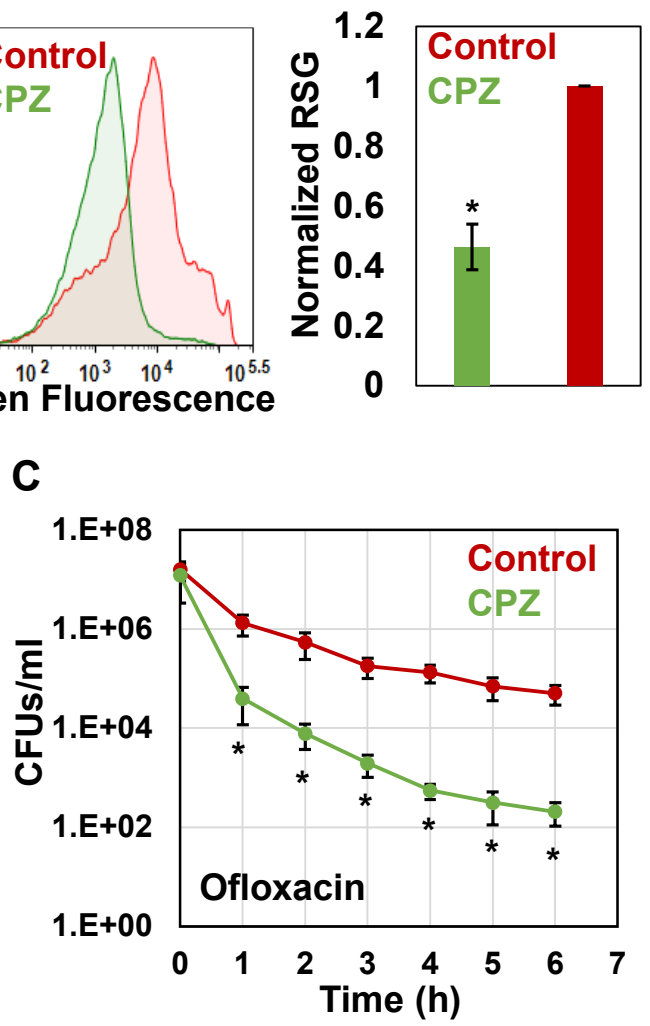

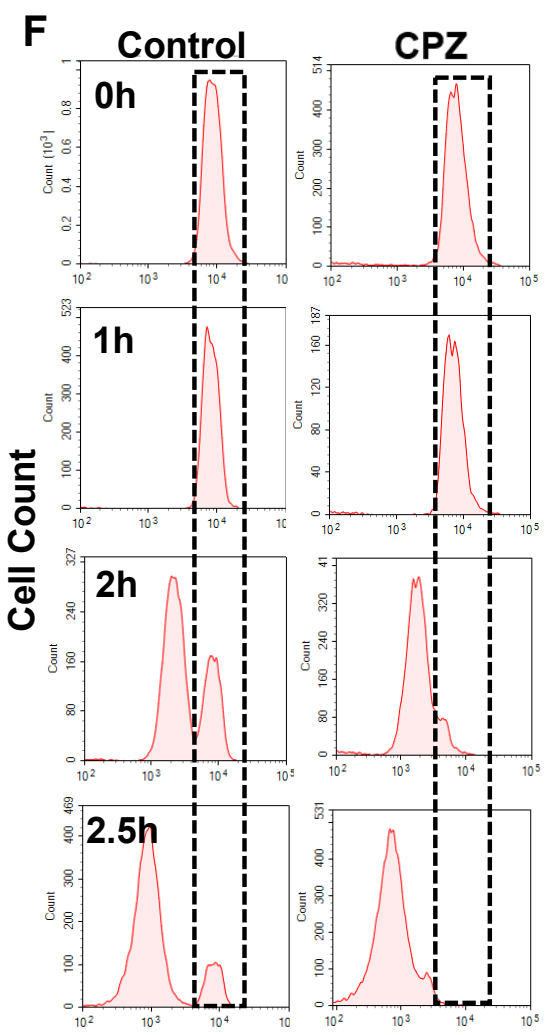

Red Fl.

Fig. 1. Chlorpromazine (CPZ) treatment reduced stationary-phase persistence, redox activities and non-growing cell formation.

(A) CPZ treatment. Early-stationary-phase cells $(\mathrm{t}=5 \mathrm{~h})$ were treated with $0.25-\mathrm{mM} \mathrm{CPZ}$ or left untreated (control); cells in late-stationary phase $(\mathrm{t}=24 \mathrm{~h})$ were then washed to remove inhibitors to measure persister and non-growing cell levels as well as cellular redox activities. Cell growth was monitored with $\mathrm{OD}_{600}$ measurements.

(B-C) Persister levels of CPZ-treated cultures. Untreated or CPZ-treated cells in late-stationary phase $(\mathrm{t}=24 \mathrm{~h})$ were washed to remove chemicals and resuspended in fresh media with antibiotics

591 for persister quantitation (Number of biological replicates, $\mathrm{N}=6$ ).

(D-E) RSG staining of CPZ-treated or untreated late-stationary-phase cells. Untreated or CPZ-

treated cells in late-stationary phase $(\mathrm{t}=24 \mathrm{~h})$ were washed to remove the chemicals, and resuspended in PBS to stain with $\mathrm{RSG}(\mathrm{N}=6)$. 
595 (F) Non-growing cell levels in CPZ-treated cells. Early-stationary-phase cells (harboring an IPTG

596 inducible mCherry expression cassette) were treated with $0.25-\mathrm{mM} \mathrm{CPZ} \mathrm{or} \mathrm{left} \mathrm{untreated} \mathrm{(control)}$

597 at $\mathrm{t}=5 \mathrm{~h}$ in the presence of IPTG; cells in late-stationary phase $(\mathrm{t}=24 \mathrm{~h})$ were washed to remove the

598 chemicals and diluted in fresh media without inducer. Division at the single-cell level was

599 monitored by flow cytometry during exponential-growth phase. A representative biological

600 replicate is shown here. All 3 biological replicates consistently resulted in similar trends.

601 *: Statistical significance between control (untreated) vs. CPZ treatment group at indicated time

602 points or conditions ( $\mathrm{P}<0.05$, two-tailed t-tests with unequal variances). 
A

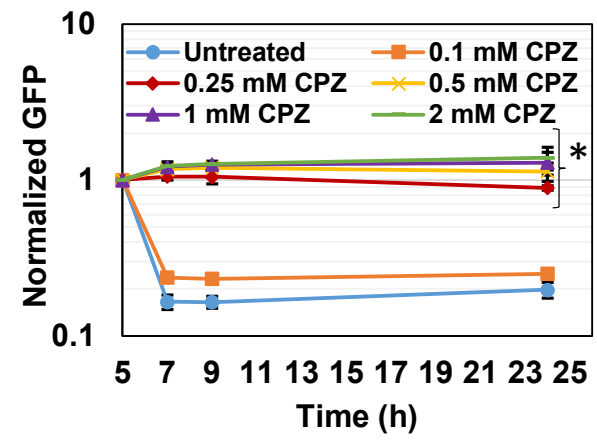

C

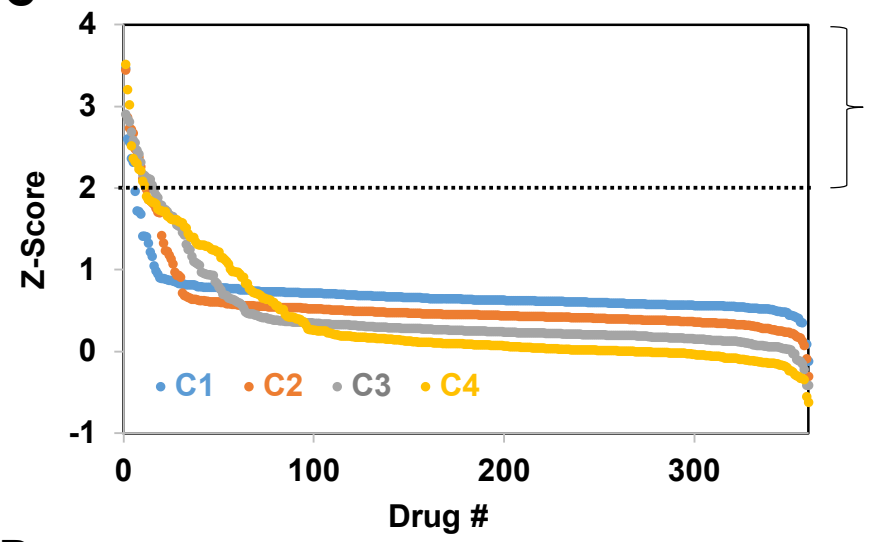

D
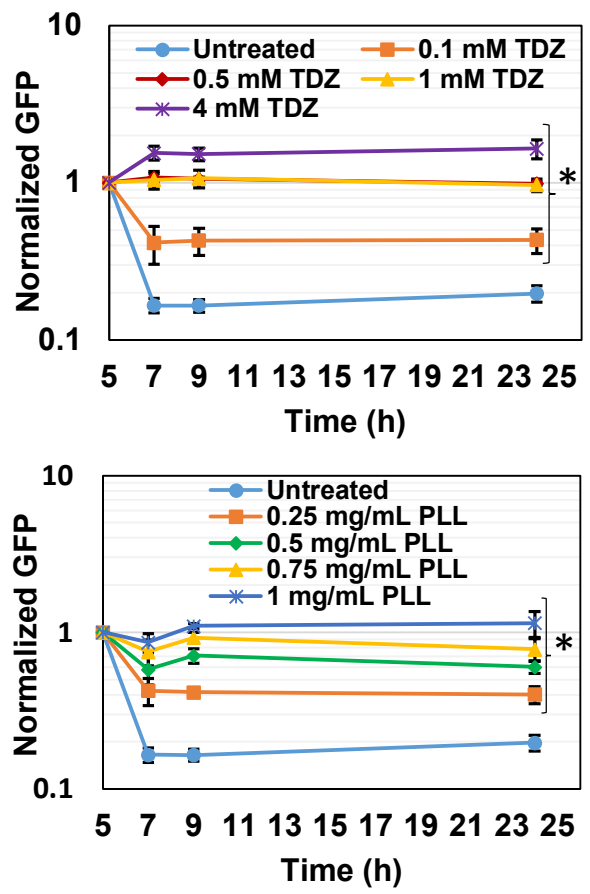

B
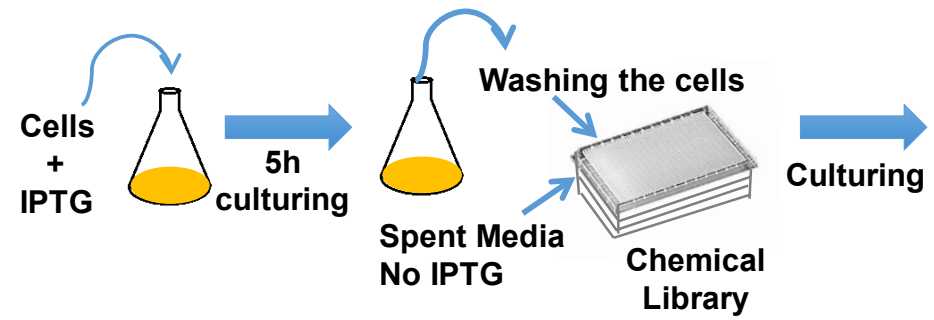

Polymyxin B (PMXB)

CCCP and FCCP

Poly-L-lysine hydrochloride (PLL)

Amitriptyline hydrochloride

Thioridazine (TDZ)

Trifluoperazine (TFP)

Triclosan

Protamine sulfate

Promethazine

Chlorpromazine hydrochloride

Dodecyltrimethyl ammonium bromide
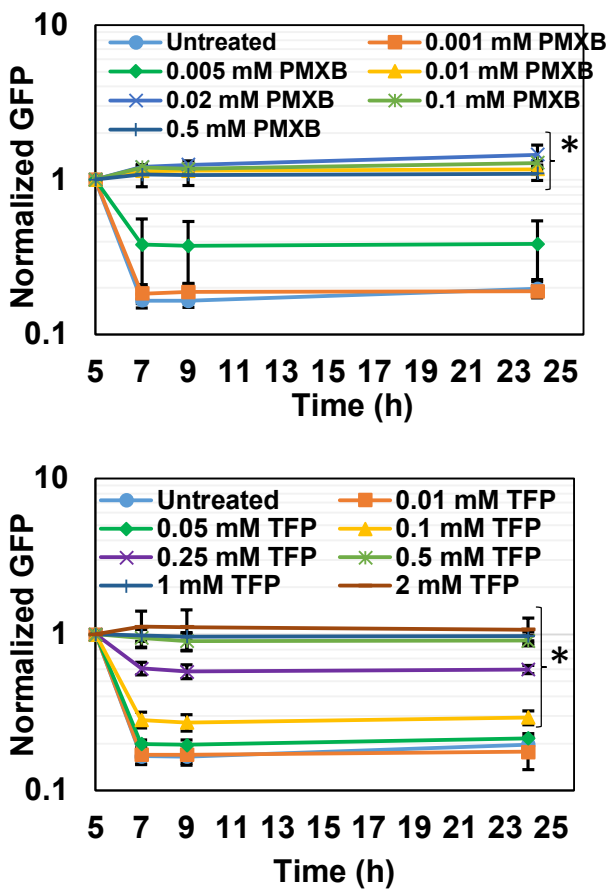

E

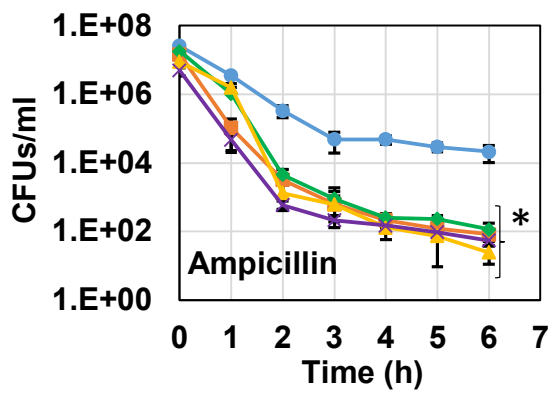

$\rightarrow-$ Untreated $\quad-0.5 \mathrm{mM}$ TFP $\multimap 0.75 \mathrm{mg} / \mathrm{mL}$ PLL $\rightarrow 0.5 \mathrm{mM} \mathrm{TDZ}$ $\because 0.01 \mathrm{mM}$ PMXB

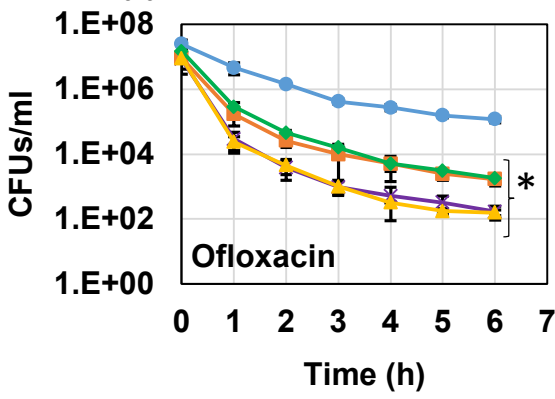

Fig. 2. High-throughput drug screening detected chemical compounds that inhibit persistence. 
(A) Inhibition of GFP degradation with CPZ treatment at indicated concentrations. Cells expressing pQE-80Lgfp-ssrA were grown to stationary phase $(\mathrm{t}=5 \mathrm{~h})$ in the presence of IPTG (inducer) and then re-suspended in a filter-sterilized spent medium (without inducer and obtained from the cultures grown under identical conditions) and immediately treated with CPZ to inhibit cell metabolism and protein degradation. Green fluorescence levels were measured and normalized to their initial levels $(\mathrm{t}=5 \mathrm{~h}$, before CPZ treatment) to determine GFP degradation. Background fluorescence was determined using cells with empty vectors $(\mathrm{N}=3)$.

(B) High-throughput drug screening approach to identify chemical compounds that inhibit GFP degradation. Stationary-phase bacterial cells expressing ssrA-tagged GFP were re-suspended in spent medium, without inducer, transferred to 96-well PM plates containing the chemical library, covered with sterile, oxygen-permeable sealing membranes, and cultured in a shaker for $4 \mathrm{~h}$. GFP measurements taken at $4 \mathrm{~h}$ were normalized to those taken at $0 \mathrm{~h}$ (after transferring the cells to plates).

(C) The Z-scores calculated for the chemical compounds at four different concentrations $\left(\mathrm{C}_{4}>\mathrm{C}_{3}>\right.$ $\mathrm{C}_{2}>\mathrm{C}_{1}$ ). Note that these concentrations were not disclosed by Biolog, Inc. The initial hits tabulated were selected among the chemicals that have $Z$-scores $\geq 2$ with at least two different concentrations. (D-E) Inhibition of GFP degradation and persistence by the identified drugs. The selected hits were analyzed in depth at various concentrations to select the drugs that can reduce GFP degradation and persistence without affecting the $E$. coli cell viability. Cells were treated with these drugs at early stationary phase $(\mathrm{t}=5 \mathrm{~h})$ at indicated concentrations, and then, GFP measurements were performed at indicated time points. Persister assays were performed at late stationary phase $(\mathrm{t}=24 \mathrm{~h})(\mathrm{N}=3)$.

*: Statistical significance between drug-treated vs. untreated cultures at last three time points ( $\mathrm{P}<0.05$, two-tailed $\mathrm{t}$-tests with unequal variances).

CPZ: Chlorpromazine; PMXB: Polymyxin B; PLL: Poly-L-lysine; TDZ: Thioridazine; TFP: Trifluoperazine. 
A
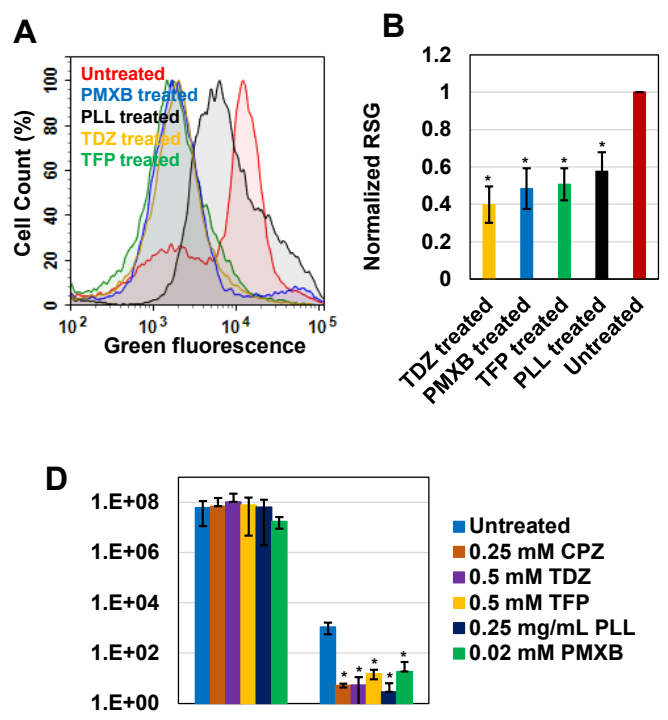

631

\section{formation.} are the same as those provided in panels A-B. and persistence, respectively $(\mathrm{N}=6)$.
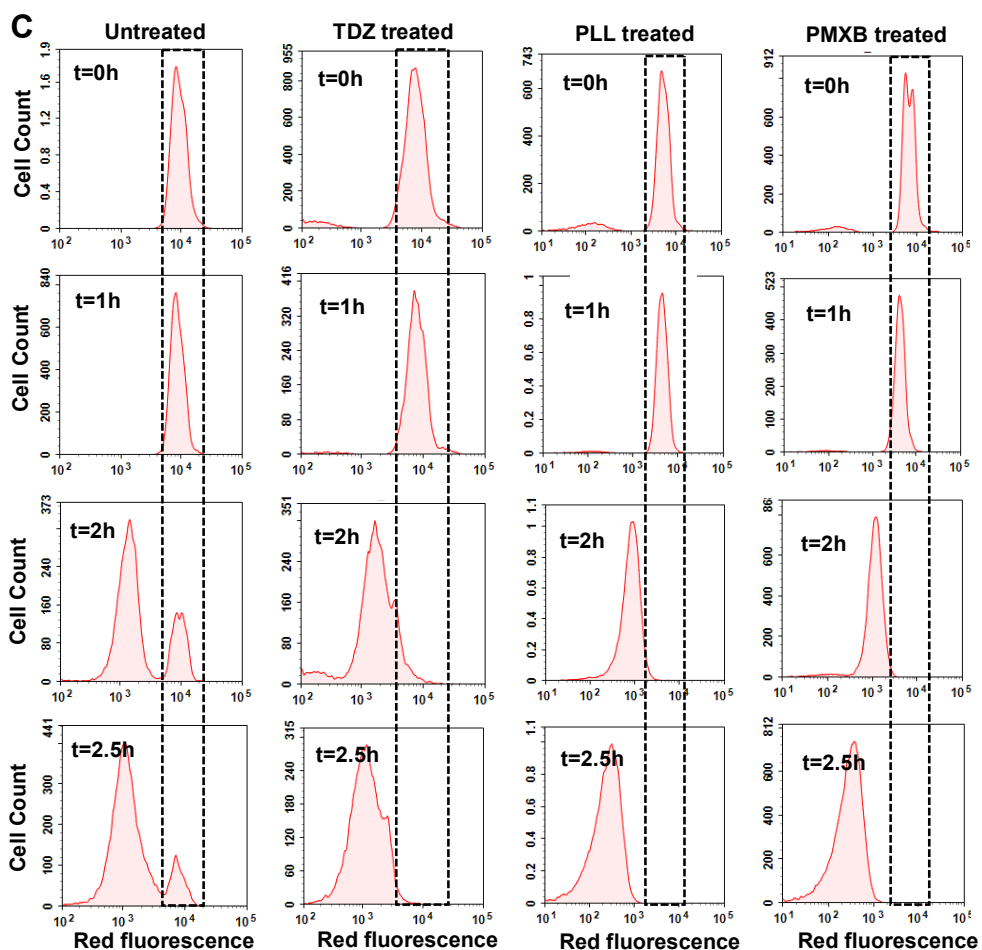

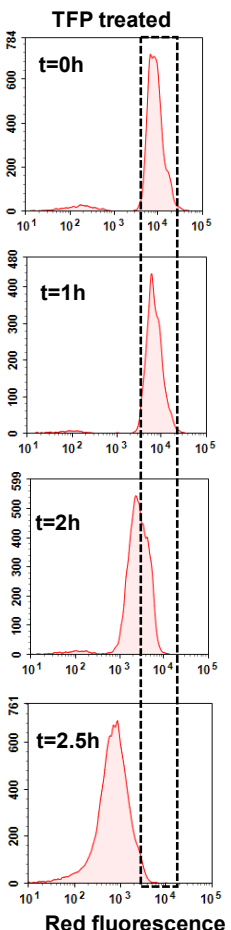

Fig. 3. Drug treatments reduced stationary-phase redox activities and non-growing cell

(A-B) RSG staining of drug-treated or untreated late-stationary-phase E. coli cells. Cells were treated with the drugs at early stationary phase $(\mathrm{t}=5 \mathrm{~h})$, and RSG staining was performed at latestationary phase $(\mathrm{t}=24 \mathrm{~h})$. Drug concentrations: $0.5 \mathrm{mM}$ Thioridazine (TDZ); $0.75 \mathrm{mg} / \mathrm{ml}$ Poly-Llysine (PLL); 0.01 mM Polymyxin B (PMXB); 0.5 mM Trifluoperazine (TFP) (N=6).

(C) Non-growing cell levels in drug-treated E. coli cultures. A representative biological replicate is shown here. All 3 biological replicates consistently resulted in similar trends. Drug concentrations

(D) Persister levels in P. aeruginosa cultures treated with the chemical hits. Early-stationary-phase cells $(\mathrm{t}=5 \mathrm{~h})$ were treated with the selected drugs or left untreated (control); cells in late stationary phase were then washed to remove inhibitors and re-suspended in fresh media with ofloxacin (effective for P. aeruginosa) (58) for persister assays. Cells were plated for CFU enumeration before and after the ofloxacin treatments to assess the effects of drugs on P. aeruginosa cell viability 
bioRxiv preprint doi: https://doi.org/10.1101/737320; this version posted September 10, 2019. The copyright holder for this preprint (which was not certified by peer review) is the author/funder, who has granted bioRxiv a license to display the preprint in perpetuity. It is made available under aCC-BY-NC-ND 4.0 International license.

647 *: Statistical significance between drug-treated vs. untreated cultures $(\mathrm{P}<0.05$, two-tailed t-tests

648 with unequal variances).

649

650

651

652

653

654

655

656

657

658

659

660

661

662

663 